\title{
Toracotomías posteriores a pleurotomía mínima en el manejo del trauma penetrante torácico*
} Requirement of thoracotomy in penetrating chest trauma

\author{
Dr. MAURICIO CANCINO Q. ${ }^{1}$ Ints. ALEJANDRA PARRA C. ${ }^{2}$, DANIELA KAWAS G. ${ }^{2}$, \\ ALEJANDRA CRUZAT S. ${ }^{2}$, MARÍA JOSÉ ORTIZ B. ${ }^{2}$, Dr. ALEJANDRO RODRÍGUEZ N. ${ }^{3}$ \\ ${ }^{1}$ Hospital Dr. Eduardo Pereira, Valparaíso, Chile. ${ }^{2}$ Internas Cátedra de Cirugía, Universidad de Valparaíso, Chile. \\ ${ }^{3}$ Hospital Dr. Carlos Van Buren, Valparaíso, Chile.
}

\begin{abstract}
RESUMEN
El trauma en Chile es la primera causa de muerte en pacientes menores de 45 años, siendo el trauma penetrante torácico especialmente importante debido a su alta frecuencia en nuestro medio. Este es un estudio observacional censal descriptivo, en el cual se revisaron los libros de protocolo operatorio del Hospital Carlos Van Buren de Valparaíso, desde Diciembre de 1997 hasta enero del 2006, obteniendo el total de pacientes ingresados por trauma penetrante torácico a los que se les realizó pleurotomía mínima, siendo estos 203 pacientes. Se revisaron las fichas con el objetivo de determinar cuántos pacientes respondieron adecuadamente sólo con pleurotomía mínima y cuántos requirieron toracotomía de urgencia - electiva. Los resultados se describen en porcentajes obteniendo que del total de pacientes $92,6 \%$ corresponden a hombres y 7,39\% corresponden a mujeres. De los 203 pacientes estudiados 10,8\% requirieron toracotomía y $89,2 \%$ evolucionaron favorablemente sólo con pleurotomía mínima. La causa más frecuente de toracotomía fue el hemotórax masivo $(50 \%)$, el cual fue manejado satisfactoriamente, siendo diagnosticado y tratado precozmente. El trauma penetrante torácico es más frecuente en el sexo masculino, sobre todo jóvenes, siendo la mayoría resueltos exitosamente con la pleurotomía mínima en la Unidad de Emergencias.
\end{abstract}

PALABRAS CLAVE: Toracotomía, toracostomía, neumotorax, hemoneumotorax, hemotórax.

\section{SUMMARY}

Background: Trauma is the first cause of death among subjects of less than 45 years of age in Chile. Penetrating chest trauma is common. Aim: To assess the need for thoracotomy among patients with penetrating chest trauma. Material and methods: Retrospective review of operative protocols of all tube thoracostomies performed in a General Hospital from 1997 to 2006. Results: In the study period, 275 protocols of tube thoracostomy were found and 72 had to be discarded. Therefore, 203 patients, aged 15 to 80 years (188 males), were included in the study. Eleven percent of patients required a surgical thoracotomy and the rest only required the tube thoracostomy. The most common reason to perform a thoracotomy was massive hemothorax in $50 \%$ of cases. Conclusions: Most cases of penetrating chest trauma occur in men and can be successfully treated with a tube thoracostomy.

\section{KEY WORDS: Chest trauma, tube thoracostomy, thoracotomy.}

"Recibido el 12 de Enero de 2007 y aceptado para publicación el 6 de mayo de 2007.

Correspondencia: Dr. Alejandra Parra C.

Avenida Camino del Sol 2300 casa K1, Jardines de Paso Hondo IV. Viña del Mar, Chile e mail: aleparra@gmail.com 


\section{INTRODUCCIÓN}

El trauma en Chile, desde el año 1977, es la tercera causa de muerte en la población general, precedido por las enfermedades cardiovasculares y oncológicas; siendo la primera causa de muerte en la población entre 15 y 45 años ${ }^{1}$.

El trauma penetrante torácico por arma blanca, tiene gran relevancia debido a su alta frecuencia en nuestro medio, siendo la primera causa de trauma torácico en los últimos años en nuestra ciudad ${ }^{2}$. Este tipo de traumatismo ocurre como consecuencia de una agresión, cuyas lesiones incluyen pared torácica, pulmones, corazón, grandes vasos y diafragma, y por tanto comprometen en mayor o menor grado la función respiratoria, circulatoria y cardíaca.

Estos son pacientes graves, que exigen un manejo precoz y efectivo ya que son causa de severas lesiones de órganos intratorácicos que pueden ser rápidamente fatales; aquí toma especial importancia la pleurotomía mínima como manejo de urgencia ${ }^{3}$.

En este trabajo se pretende conocer el porcentaje de toracotomías posteriores a pleurotomía mínima por trauma penetrante torácico en nuestro medio.

\section{MATERIAL Y MÉTODO}

Se trata de un estudio observacional censal descriptivo ${ }^{4}$; en el cual se revisaron los libros de protocolo operatorio del Hospital Carlos Van Buren desde Diciembre de 1997 hasta enero del 2006. Nuestro único criterio de inclusión fue:

- Pleurotomía mínima por traumatismo penetrante de tórax, ya sea por arma blanca o de fuego.

Se revisaron en total 275 protocolos, obteniéndose los datos de fecha, nombre, edad, sexo, diagnóstico pre y post pleurotomía y técnica utilizada.

En el Hospital Carlos Van Buren, se realizan dos técnicas de pleurotomía mínima:

- Con Argylle: sin exploración digital.

- Con pinza Kelly: con exploración digital.

La inserción del tubo pleural se realiza según la técnica descrita en ATLS $^{5}$.

De los 275 protocolos obtenidos fueron descartados $72(26,1 \%)$ pacientes por las siguientes causas:

- Nombre incompleto o ininteligible en protocolo:

- Sin número de ficha en el registro de archivos:

- Fichas no encontradas:

- Trauma cerrado:

- Derivado al Hospital Gustavo Fricke:
Nuestra cifra final fue de 203 pacientes.

Se revisaron las fichas de dichos pacientes en la oficina de archivos del hospital.

En caso de haberse realizado la toracotomía se anotó de dicho protocolo la fecha y los diagnósticos pre y postoperatorios. También se rescató de la ficha el número de días de estadía en la unidad.

Se recopilaron los datos, obteniendo los siguientes resultados.

\section{RESULTADOS}

La edad promedio de los 203 pacientes a los que se le realizó pleurotomía mínima fue de 26,5 años en un rango que va entre 15 a 80 años.

Del total de pacientes $92,6 \%$ (188) corresponden a hombres y $7,39 \%$ (15) corresponden a mujeres (Figura 1).

Del total de pleurotomías mínimas realizadas, un $21,18 \%$ (43) fueron con diagnóstico de hemotórax, 42,86\% (87) de neumotórax y 36\% (73) de hemoneumotorax (Figura 2).

Acerca de las técnicas de pleurotomía mínima $27 \%$ (55) se realizaron con técnica sin exploración, $52,22 \%$ (106) se realizaron con técnica con exploración y en $20,7 \%$ (42) no se describía en el protocolo operatorio.

De los 203 pacientes estudiados 10,8\% (22) requirieron toracotomía y $89,2 \%$ (181) evolucionaron favorablemente sólo con pleurotomía mínima. (Figura 3)

En el grupo de hombres el 10,10\% (19) requirió toracotomía, mientras que en el grupo de mujeres la cifra fue de un 20\% (3) (Figura 4).

Del total de toracotomías, $36,4 \%$ (8) tenían diagnóstico postpleurotomía de hemotórax, 63,6\% (14) por hemoneumotórax; ninguno de los pacientes con diagnóstico de neumotórax requirió toracotomía.

Del total de toracotomías 31,8\% (7) fue posterior a una pleurotomía con técnica sin exploración,

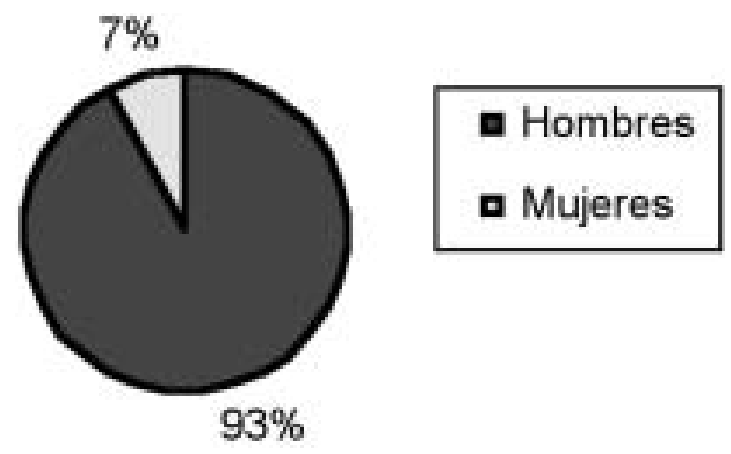

Figura 1. Distribución por sexo del total de pacientes. 


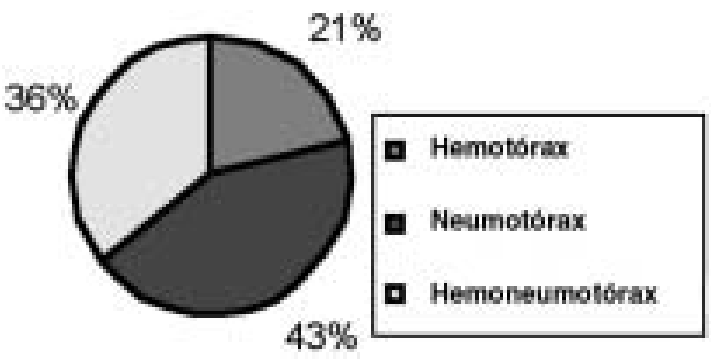

Figura 2. Diagnósticos postpleurotomía.

$59,1 \%$ (13) fue posterior a técnica con exploración y en $9 \%^{2}$ no estaba descrito en el protocolo.

Acerca de las causas de las toracotomías $50 \%{ }^{11}$ corresponden a hemotórax masivo, 27,3\% ${ }^{6}$ fueron por hemotórax retenido, 9\% (2) corresponden a empiema, 9\% (2) por hemotórax organizado y $4,5 \%$ (1) por taponamiento cardíaco (Figura 5).

El promedio de días transcurridos desde la pleurotomía mínima a la toracotomía fue de 4,54 días, en un rango que va desde el mismo día de ingreso a UEA hasta 30 días post pleurotomía. La mortalidad en el total de pacientes analizados fue de un $0 \%$.

\section{DISCUSIÓN}

Se puede apreciar que el promedio de edad del trauma penetrante torácico es de 26,5 años lo cual concuerda con lo descrito en la literatura respecto a la edad de mayor frecuencia de traumatismo torácico, ${ }^{5-9}$ significando un gran impacto social al afectar a una población previamente sana y en plena actividad productiva.

La mayoría de los pacientes que ingresan por trauma penetrante torácico son hombres $(92,6 \%)$, en una relación de 12,5:1; concordando con datos de referencia internacional ${ }^{5-9}$, al ser los más expuestos a agresiones por terceros.

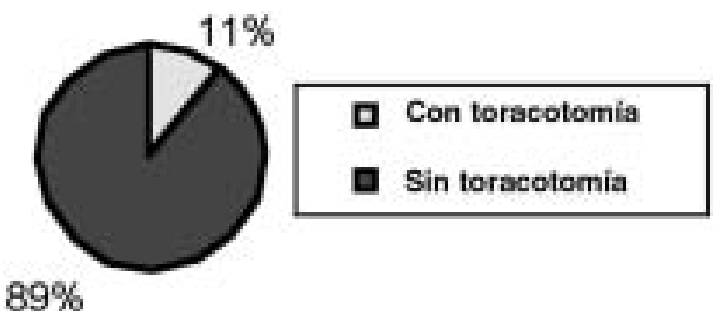

Figura 3. Porcentaje de toracotomía del total de pacientes.

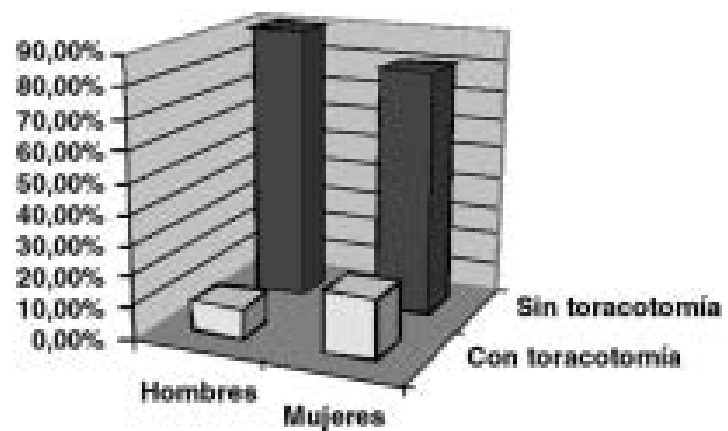

Figura 4. Porcentaje de toracotomías según sexo.

La causa más frecuente de pleurotomía fue el neumotórax (43\%). Este dato coincide con los encontrados en un trabajo local que estudiaba tanto trauma torácico abierto como cerrado ${ }^{2}$, pero se contradice con estudios de otros centros en que el neumotórax se encuentra en proporciones menores $^{6,8,9}$.

Respecto a las técnicas de pleurotomía mínima, la mayoría fue con exploración digital (52,2\%); lo que coincide con el actual manejo en nuestro medio, establecido hace 2 años.

Del total de pacientes, un 10,8\% requirió de toracotomía, lo cual está en un rango aceptable en comparación con un 15\% o más descrito en el Manual de ATLS y literatura1,2,5,9.

A pesar de que el trauma penetrante es más frecuente en el sexo masculino, hay un mayor porcentaje de toracotomías en el sexo femenino. El total de éstas (3 casos), fueron de urgencias, debido a hemotórax masivo; esto se podría explicar por un menor desarrollo muscular de la pared torácica femenina que expone más fácilmente las estructuras susceptibles de sangrar en forma masiva, frente a una agresión por arma blanca.

Respecto a las causas de toracotomía, se observa que la mitad son debido a hemotórax masi-

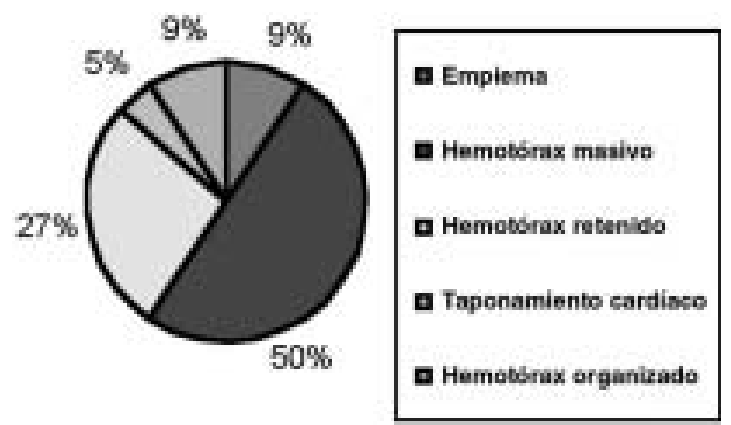

Figura 5. Causas de toracotomía. 
vo, por lo tanto corresponden a una indicación de cirugía inmediata, mientras la otra mitad son realizadas posteriormente debido a complicaciones, lo cual concuerda con lo encontrado en la literatu$\mathrm{ra}^{2,7,9}$.

Acerca del neumotórax, el manejo con pleurotomía mínima es adecuado, no habiendo complicaciones que requieran de una toracotomía.

Del número total de toracotomías, sólo 9\% (2) fue debido a una complicación infecciosa. Es relevante mencionar que estos 2 casos corresponden a pacientes que se fugaron antes de resolver su ocupación pleural y reingresaron con el cuadro séptico instalado.

El 27\% de las toracotomías fue por hemotórax retenido, lo que debe motivar a un estudio de los factores involucrados.

El promedio de 4,5 días desde la pleurotomía mínima a la toracotomía es inferior al encontrado en la literatura internacional (6 a 10 días) ${ }^{9}$.

El manejo del hemotórax masivo en urgencias demuestra ser satisfactorio, al ser diagnosticado y resuelto precozmente, teniendo una evolución favorable.

La mayoría de los traumatismos penetrantes torácicos se resuelven con el manejo inicial en la Unidad de Emergencias.

\section{REFERENCIAS}

1. Bianchi V, Parra J. Trauma: una nueva epidemia. En: Carvajal C, Uribe M, Cavallieri S. Trauma: Manejo Avanzado. Santiago: Editor Dr Antonio Yuri, 1997; 35-39.

2. Valenzuela M, Cancino P, Cabezas F, Donoso G, De la Torre I. Experiencia en traumatismo torácico, Hospital Valparaíso. Rev Chil Cir 2003; 55: 449-453.

3. Chavez C, Raffo A, Elgueta C. Trauma penetrante torácico, resultados de una conducta terapéutica selectiva. Rev Chil Cir 1989; 41: 344-348.

4. Manterola C. Estrategias de investigación. 1ra Parte. Estudios descriptivos. Rev Chil Cir 2001; 53: 229-233.

5. Capítulo 4: Traumatismo torácico. En: American College of Surgeons. Advanced Trauma Life Support Program. Chicago, IL, 2002; 93-116.

6. Fernandez N, Perroti P, Sandrigo S, Giroldi K. Manejo del espacio pleural en el trauma torácico. Rev Posgrado VI Cátedra Med 2005; 142: 10-15.

7. Bello N, Borquez P, Guridi R, Baeza A, Lilayu D. Perfil y manejo del trauma torácico en un Hospital Regional. Rev Chil Cir 2005; 57: 393-396.

8. Solis S, Berges R, Fernandez M, Mendez R. Toracotomías de Urgencia y Emergencia. Rev Cubana Cir 2000; 39: 47-51.

9. Oettinger R, Soto S. Pleurotomías en el Hospital Clínico Regional de Valdivia, generalidades y estudio analítico del período Junio 1995 - Junio 2000. Cuad Cir 2001; 15: 29-34. 